\title{
On Stanley's Partition Function
}

\author{
William Y. C. Chen ${ }^{1}$, Kathy Q. Ji ${ }^{2}$, and Albert J. W. Zhu ${ }^{3}$ \\ Center for Combinatorics, LPMC-TJKLC \\ Nankai University, Tianjin 300071, P.R. China \\ ${ }^{1}$ chen@nankai.edu.cn, ${ }^{2}$ ji@nankai.edu.cn, ${ }^{3}$ zjw@cfc.nankai.edu.cn \\ Submitted: Jun 12, 2010; Accepted: Aug 19, 2010; Published: Sep 1, 2010 \\ Mathematics Subject Classification: 05A17
}

\begin{abstract}
Stanley defined a partition function $t(n)$ as the number of partitions $\lambda$ of $n$ such that the number of odd parts of $\lambda$ is congruent to the number of odd parts of the conjugate partition $\lambda^{\prime}$ modulo 4 . We show that $t(n)$ equals the number of partitions of $n$ with an even number of hooks of even length. We derive a closed-form formula for the generating function for the numbers $p(n)-t(n)$. As a consequence, we see that $t(n)$ has the same parity as the ordinary partition function $p(n)$. A simple combinatorial explanation of this fact is also provided.
\end{abstract}

\section{Introduction}

This note is concerned with the partition function $t(n)$ introduced by Stanley $[8,9]$. We shall give a combinatorial interpretation of $t(n)$ in terms of hook lengths and shall prove that $t(n)$ and the partition function $p(n)$ have the same parity. Moreover, we compute the generating function for $p(n)-t(n)$.

We shall adopt the common notation on partitions in Andrews [1] or Andrews and Eriksson [3]. A partition $\lambda=\left(\lambda_{1}, \lambda_{2}, \lambda_{3}, \ldots, \lambda_{r}\right)$ of a nonnegative integer $n$ is a nonincreasing sequence of nonnegative integers such that the sum of the components $\lambda_{i}$ equals $n$. A part is meant to be a positive component, and the number of parts of $\lambda$ is called the length, denoted $l(\lambda)$. The conjugate partition of $\lambda$ is defined by $\lambda^{\prime}=\left(\lambda_{1}^{\prime}, \lambda_{2}^{\prime}, \ldots, \lambda_{t}^{\prime}\right)$, where $\lambda_{i}^{\prime}(1 \leqslant i \leqslant t, t=l(\lambda))$ is the number of parts in $\left(\lambda_{1}, \lambda_{2}, \ldots, \lambda_{r}\right)$ which are greater than or equal to $i$. The number of odd parts in $\lambda=\left(\lambda_{1}, \lambda_{2}, \ldots, \lambda_{r}\right)$ is denoted by $\mathcal{O}(\lambda)$.

For $|q|<1$, the $q$-shifted factorial is defined by

$$
(a ; q)_{n}=(1-a)(1-a q) \cdots\left(1-a q^{n-1}\right), \quad n \geqslant 1,
$$

and

$$
(a ; q)_{\infty}=(1-a)(1-a q)\left(1-a q^{2}\right) \cdots
$$


see Gasper and Rahman [5].

Stanley $[8,9]$ introduced the partition function $t(n)$ as the number of partitions $\lambda$ of $n$ such that $\mathcal{O}(\lambda) \equiv \mathcal{O}\left(\lambda^{\prime}\right)(\bmod 4)$, and obtained the following formula

$$
t(n)=\frac{1}{2}(p(n)+f(n)),
$$

where $p(n)$ is the number of partitions of $n$ and $f(n)$ is determined by the generating function

$$
\sum_{n=0}^{\infty} f(n) q^{n}=\prod_{i \geqslant 1} \frac{\left(1+q^{2 i-1}\right)}{\left(1-q^{4 i}\right)\left(1+q^{4 i-2}\right)^{2}} .
$$

Andrews [2] obtained the following closed-form formula for the generating function of $t(n)$

$$
\sum_{n=0}^{\infty} t(n) q^{n}=\frac{\left(q^{2} ; q^{2}\right)_{\infty}^{2}\left(q^{16} ; q^{16}\right)_{\infty}^{5}}{(q ; q)_{\infty}\left(q^{4} ; q^{4}\right)_{\infty}^{5}\left(q^{32} ; q^{32}\right)_{\infty}^{2}}
$$

He also derived the congruence relation

$$
t(5 n+4) \equiv 0(\bmod 5) .
$$

In this note, we shall consider the complementary partition function of $t(n)$, namely, the partition function $u(n)=p(n)-t(n)$, which is the number of partitions $\lambda$ of $n$ such that $\mathcal{O}(\lambda) \not \equiv \mathcal{O}\left(\lambda^{\prime}\right)(\bmod 4)$. We obtain a closed-form formula for the generating function of $u(n)$ which implies that Stanley's partition function $t(n)$ and ordinary partition function $p(n)$ have the same parity for any $n$. We also present a simple combinatorial explanation of this fact. Furthermore, we derive formulas for the generating functions for the numbers $u(4 n), u(4 n+1), u(4 n+2)$ and $u(4 n+3)$, which are analogous to the generating function formulas for the partition functions $t(4 n), t(4 n+1), t(4 n+2)$ and $t(4 n+3)$ due to Andrews [2]. In the last section, we find combinatorial interpretations for $t(n)$ and $u(n)$ in terms of hooks of even length.

\section{The generating function formula}

In this section, we shall derive a generating function formula for the partition function $u(n)=p(n)-t(n)$. The proof is similar to Andrews' proof of (1.3) for $t(n)$. As a consequence, one sees that $t(n)$ and $p(n)$ have the same parity for any nonnegative integer $n$. This fact also has a simple combinatorial interpretation. We shall also compute the generating functions for the numbers $u(4 n), u(4 n+1), u(4 n+2)$ and $u(4 n+3)$.

Theorem 2.1 We have

$$
\sum_{n=0}^{\infty} u(n) q^{n}=\frac{2 q^{2}\left(q^{2} ; q^{2}\right)_{\infty}^{2}\left(q^{8} ; q^{8}\right)_{\infty}^{2}\left(q^{32} ; q^{32}\right)_{\infty}^{2}}{(q ; q)_{\infty}\left(q^{4} ; q^{4}\right)_{\infty}^{5}\left(q^{16} ; q^{16}\right)_{\infty}}
$$


Proof. We notice that the definition of $t(n)$ implies

$$
u(n)=p(n)-t(n)=\frac{p(n)-f(n)}{2} .
$$

Hence we have

$$
\begin{aligned}
\sum_{n=0}^{\infty} u(n) q^{n} & =\frac{1}{2}\left(\frac{1}{(q ; q)_{\infty}}-\frac{\left(-q ; q^{2}\right)_{\infty}}{\left(q^{4} ; q^{4}\right)_{\infty}\left(-q^{2} ; q^{4}\right)_{\infty}^{2}}\right) \\
& =\frac{1}{2}\left(\frac{\left(-q ; q^{2}\right)_{\infty}}{\left(q^{4} ; q^{4}\right)_{\infty}\left(q^{2} ; q^{4}\right)_{\infty}^{2}}-\frac{\left(-q ; q^{2}\right)_{\infty}}{\left(q^{4} ; q^{4}\right)_{\infty}\left(-q^{2} ; q^{4}\right)_{\infty}^{2}}\right) \\
& =\frac{\left(-q ; q^{2}\right)_{\infty}}{2\left(q^{4} ; q^{4}\right)_{\infty}^{2}\left(q^{2} ; q^{4}\right)_{\infty}^{2}\left(-q^{2} ; q^{4}\right)_{\infty}^{2}}\left(\left(q^{4} ; q^{4}\right)_{\infty}\left(-q^{2} ; q^{4}\right)_{\infty}^{2}-\left(q^{4} ; q^{4}\right)_{\infty}\left(q^{2} ; q^{4}\right)_{\infty}^{2}\right) .
\end{aligned}
$$

Using Jacobi's triple product identity [4, p.10]

$$
\sum_{n=-\infty}^{\infty} z^{n} q^{n^{2}}=\left(-z q ; q^{2}\right)_{\infty}\left(-q / z ; q^{2}\right)_{\infty}\left(q^{2} ; q^{2}\right)_{\infty}
$$

we see that

$$
\left(q^{4} ; q^{4}\right)_{\infty}\left(-q^{2} ; q^{4}\right)_{\infty}^{2}=\sum_{n=-\infty}^{\infty} q^{2 n^{2}}
$$

and

$$
\left(q^{4} ; q^{4}\right)_{\infty}\left(q^{2} ; q^{4}\right)_{\infty}^{2}=\sum_{n=-\infty}^{\infty}(-1)^{n} q^{2 n^{2}}
$$

Clearly,

$$
\sum_{n=-\infty}^{\infty} q^{2 n^{2}}-\sum_{n=-\infty}^{\infty}(-1)^{n} q^{2 n^{2}}=2 \sum_{n=-\infty}^{\infty} q^{2(2 n+1)^{2}}
$$

Thus we obtain

$$
\begin{aligned}
\sum_{n=0}^{\infty} u(q) q^{n} & =\frac{\left(-q ; q^{2}\right)_{\infty}}{\left(q^{4} ; q^{4}\right)_{\infty}^{2}\left(q^{2} ; q^{4}\right)_{\infty}^{2}\left(-q^{2} ; q^{4}\right)_{\infty}^{2}} \sum_{n=-\infty}^{\infty} q^{2(2 n+1)^{2}} \\
& =\frac{q^{2}\left(-q ; q^{2}\right)_{\infty}}{\left(q^{4} ; q^{4}\right)_{\infty}^{2}\left(q^{4} ; q^{8}\right)_{\infty}^{2}} \sum_{n=-\infty}^{\infty} q^{8 n^{2}+8 n}
\end{aligned}
$$

Using Jacobi's triple product identity, we find

$$
\sum_{n=-\infty}^{\infty} q^{8 n^{2}+8 n}=\left(-q^{16} ; q^{16}\right)_{\infty}\left(-1 ; q^{16}\right)_{\infty}\left(q^{16} ; q^{16}\right)_{\infty}
$$

Observe that

$$
\left(-1 ; q^{16}\right)_{\infty}=2\left(-q^{16} ; q^{16}\right)_{\infty}
$$


In view of $(2.11)$, we get

$$
\begin{aligned}
\sum_{n=0}^{\infty} u(q) q^{n} & =\frac{2 q^{2}\left(-q^{16} ; q^{16}\right)_{\infty}\left(-q^{16} ; q^{16}\right)_{\infty}\left(-q ; q^{2}\right)_{\infty}\left(q^{16} ; q^{16}\right)_{\infty}}{\left(q^{4} ; q^{4}\right)_{\infty}^{2}\left(q^{4} ; q^{8}\right)_{\infty}^{2}} \\
& =\frac{2 q^{2}\left(q^{32} ; q^{32}\right)_{\infty}\left(-q ; q^{2}\right)_{\infty}\left(-q^{16} ; q^{16}\right)_{\infty}}{\left(q^{4} ; q^{4}\right)_{\infty}^{2}\left(q^{4} ; q^{8}\right)_{\infty}^{2}}
\end{aligned}
$$

Now,

$$
\begin{gathered}
\left(-q ; q^{2}\right)_{\infty}=\frac{\left(q^{2} ; q^{2}\right)_{\infty}^{2}}{(q ; q)_{\infty}\left(q^{4} ; q^{4}\right)_{\infty}} \\
\left(q^{4} ; q^{8}\right)_{\infty}=\frac{\left(q^{4} ; q^{4}\right)_{\infty}}{\left(q^{8} ; q^{8}\right)_{\infty}}
\end{gathered}
$$

and

$$
\left(-q^{16} ; q^{16}\right)_{\infty}=\frac{\left(q^{32} ; q^{32}\right)_{\infty}}{\left(q^{16} ; q^{16}\right)_{\infty}}
$$

Consequently,

$$
\begin{aligned}
\sum_{n=0}^{\infty} u(q) q^{n} & =\frac{2 q^{2}\left(q^{32} ; q^{32}\right)_{\infty}\left(q^{8} ; q^{8}\right)_{\infty}^{2}\left(q^{2} ; q^{2}\right)_{\infty}^{2}\left(q^{32} ; q^{32}\right)_{\infty}}{\left(q^{4} ; q^{4}\right)_{\infty}^{2}\left(q^{4} ; q^{4}\right)_{\infty}^{2}(q ; q)_{\infty}\left(q^{4} ; q^{4}\right)_{\infty}\left(q^{16} ; q^{16}\right)_{\infty}} \\
& =\frac{2 q^{2}\left(q^{2} ; q^{2}\right)_{\infty}^{2}\left(q^{8} ; q^{8}\right)_{\infty}^{2}\left(q^{32} ; q^{32}\right)_{\infty}^{2}}{(q ; q)_{\infty}\left(q^{4} ; q^{4}\right)_{\infty}^{5}\left(q^{16} ; q^{16}\right)_{\infty}}
\end{aligned}
$$

This completes the proof.

Corollary 2.2 For $n \geqslant 0$,

$$
t(n) \equiv p(n)(\bmod 2)
$$

We remark that there is a simple combinatorial explanation of the above parity property. We observe that for any partition $\lambda$ of $n$,

$$
\mathcal{O}(\lambda) \equiv \mathcal{O}\left(\lambda^{\prime}\right) \quad(\bmod 2)
$$

because we have both $\mathcal{O}(\lambda) \equiv n(\bmod 2)$ and $\mathcal{O}\left(\lambda^{\prime}\right) \equiv n(\bmod 2)$. By the definition of $u(n)$ and relation (2.17), we deduce that $u(n)$ equals the number of partitions of $n$ such that

$$
\mathcal{O}(\lambda)-\mathcal{O}\left(\lambda^{\prime}\right) \equiv 2 \quad(\bmod 4)
$$

Suppose $\lambda$ is a partition counted by $u(n)$. From (2.18) it is evident that its conjugation $\lambda^{\prime}$ is also counted by $u(n)$. Once more, from (2.18) we deduce that $\mathcal{O}(\lambda)$ and $\mathcal{O}\left(\lambda^{\prime}\right)$ are not equal, so that $\lambda$ is different from $\lambda^{\prime}$. Thus we reach the conclusion that $u(n)$ must be even, and so $t(n)$ has the same parity as $p(n)$ since $p(n)=t(n)+u(n)$.

In view of (2.6), we have the following congruence relation. 
Corollary 2.3 For $n \geqslant 0$,

$$
f(n) \equiv p(n)(\bmod 4)
$$

Theorem 2.1 enables us to derive the generating functions for $u(4 n+i)$, where $i=$ $0,1,2,3$. Andrews [2] has obtained formulas for the generating functions of $t(4 n+i)$ for $i=0,1,2,3$.

Theorem 2.4 We have

$$
\begin{aligned}
\sum_{n=0}^{\infty} u(4 n) q^{n} & =2 q^{2}\left(q^{16} ; q^{16}\right)_{\infty}\left(-q ; q^{16}\right)_{\infty}\left(-q^{15} ; q^{16}\right)_{\infty} V(q), \\
\sum_{n=0}^{\infty} u(4 n+1) q^{n} & =2 q\left(q^{16} ; q^{16}\right)_{\infty}\left(-q^{3} ; q^{16}\right)_{\infty}\left(-q^{13} ; q^{16}\right)_{\infty} V(q), \\
\sum_{n=0}^{\infty} u(4 n+2) q^{n} & =2\left(q^{16} ; q^{16}\right)_{\infty}\left(-q^{7} ; q^{16}\right)_{\infty}\left(-q^{9} ; q^{16}\right)_{\infty} V(q), \\
\sum_{n=0}^{\infty} u(4 n+3) q^{n} & =2\left(q^{16} ; q^{16}\right)_{\infty}\left(-q^{5} ; q^{16}\right)_{\infty}\left(-q^{11} ; q^{16}\right)_{\infty} V(q),
\end{aligned}
$$

where

$$
V(q)=\frac{\left(q^{2} ; q^{2}\right)_{\infty}^{2}\left(q^{8} ; q^{8}\right)_{\infty}^{2}}{(q ; q)_{\infty}^{5}\left(q^{4} ; q^{4}\right)_{\infty}}
$$

Proof. By Theorem 2.1, we find

$$
\begin{aligned}
\sum_{n=0}^{\infty} u(n) q^{n} & =\frac{2 q^{2}\left(q^{2} ; q^{2}\right)_{\infty}^{2}}{(q ; q)_{\infty}} V\left(q^{4}\right) \\
& =\frac{2 q^{2}\left(q^{2} ; q^{2}\right)_{\infty}}{\left(q ; q^{2}\right)_{\infty}} V\left(q^{4}\right)
\end{aligned}
$$

Since

$$
\frac{1}{\left(q ; q^{2}\right)_{\infty}}=(-q ; q)_{\infty}
$$

and

$$
\left(q^{2} ; q^{2}\right)_{\infty}=(q ; q)_{\infty}(-q ; q)_{\infty}
$$

we have

$$
\begin{aligned}
\sum_{n=0}^{\infty} u(n) q^{n} & =2 q^{2}(q ; q)_{\infty}(-q ; q)_{\infty}(-q ; q)_{\infty} V\left(q^{4}\right) \\
& =q^{2}(q ; q)_{\infty}(-1 ; q)_{\infty}(-q ; q)_{\infty} V\left(q^{4}\right) .
\end{aligned}
$$


Using Jacobi's triple product identity, we get

$$
(q ; q)_{\infty}(-1 ; q)_{\infty}(-q ; q)_{\infty}=\sum_{n=-\infty}^{\infty} q^{\frac{n(n+1)}{2}} .
$$

Thus we have

$$
\sum_{n=0}^{\infty} u(n) q^{n}=q^{2} \sum_{n=-\infty}^{\infty} q^{\frac{n(n+1)}{2}} V\left(q^{4}\right)=2 q^{2} \sum_{n=0}^{\infty} q^{\frac{n(n+1)}{2}} V\left(q^{4}\right) .
$$

It is easy to check that

$$
\sum_{n=0}^{\infty} q^{\frac{n(n+1)}{2}}=\sum_{n=-\infty}^{\infty} q^{2 n^{2}-n}
$$

In virtue of (2.22), we get

$$
\begin{aligned}
\sum_{n=0}^{\infty} u(n) q^{n} & =2 q^{2} \sum_{n=-\infty}^{\infty} q^{2 n^{2}-n} V\left(q^{4}\right) \\
& =2 q^{2} \sum_{i=0}^{3} \sum_{k=-\infty}^{\infty} q^{2(4 k+i)^{2}-(4 k+i)} V\left(q^{4}\right)
\end{aligned}
$$

For $i=0$, extracting the terms of the form $q^{4 j+2}$ in (2.24) for any integer $j$, we obtain

$$
\sum_{n=0}^{\infty} u(4 n+2) q^{4 n+2}=2 q^{2} \sum_{j=-\infty}^{\infty} q^{32 j^{2}-4 j} V\left(q^{4}\right)
$$

Again, Jacobi's triple product identity gives

$$
\sum_{j=-\infty}^{\infty} q^{32 j^{2}-4 j}=\left(q^{64} ; q^{64}\right)_{\infty}\left(-q^{28} ; q^{64}\right)_{\infty}\left(-q^{36} ; q^{64}\right)_{\infty}
$$

Hence we get

$$
\sum_{n=0}^{\infty} u(4 n+2) q^{4 n+2}=2 q^{2}\left(q^{64} ; q^{64}\right)_{\infty}\left(-q^{28} ; q^{64}\right)_{\infty}\left(-q^{36} ; q^{64}\right)_{\infty} V\left(q^{4}\right)
$$

which simplifies to

$$
\sum_{n=0}^{\infty} u(4 n+2) q^{n}=2\left(q^{16} ; q^{16}\right)_{\infty}\left(-q^{7} ; q^{16}\right)_{\infty}\left(-q^{9} ; q^{16}\right)_{\infty} V(q)
$$

The remaining cases can be verified using similar arguments. This completes the proof. 


\section{Combinatorial interpretations for $t(n)$ and $u(n)$}

In [8, Proposition 3.1], Stanley found three partition statistics that have the same parity as $\left(\mathcal{O}(\lambda)-\mathcal{O}\left(\lambda^{\prime}\right)\right) / 2$, and gave several combinatorial interpretations for $t(n)$. We shall present combinatorial interpretations of partition functions $t(n)$ and $u(n)$ in terms of the number of hooks of even length. For the definition of hook lengths, see Stanley [7, p. 373]. A hook of even length is called an even hook. The following theorem shows that the number of even hooks has the same parity as $\left(\mathcal{O}(\lambda)-\mathcal{O}\left(\lambda^{\prime}\right)\right) / 2$.

Theorem 3.1 For any partition $\lambda$ of $n, \mathcal{O}(\lambda) \equiv \mathcal{O}\left(\lambda^{\prime}\right)(\bmod 4)$ if and only if $\lambda$ has an even number of even hooks.

Proof. We use induction on $n$. It is clear that Theorem 3.1 holds for $n=1$. Suppose that it is true for all partitions of $n$. We aim to show that the conclusion also holds for all partitions of $n+1$. Let $\lambda$ be a partition of $n+1$ and $v=(i, j)$ be any inner corner of the Young diagram of $\lambda$, that is, the removal of the square $v$ gives a Young diagram of a partition of $n$. Let $\lambda^{-}$denote the partition obtained by removing the square $v$ from the Young diagram of $\lambda$. We use $H_{e}(\lambda)$ to denote the number of squares with even hooks in the Young diagram of $\lambda$. We claim that

$$
H_{e}(\lambda) \equiv H_{e}\left(\lambda^{-}\right) \quad(\bmod 2) \quad \text { if and only if } \quad \lambda_{i} \equiv \lambda_{j}^{\prime} \quad(\bmod 2)
$$

Let $\mathcal{T}(\lambda, v)$ denote the set of all squares in the Young diagram of $\lambda$ which are in the same row as $v$ or in the same column as $v$. After removing the square $v$ from the Young diagram of $\lambda$, the hook lengths of the squares in $\mathcal{T}(\lambda, v)$ decrease by one. Meanwhile, the hook lengths of other squares remain the same. Furthermore, if $\lambda_{i}$ and $\lambda_{j}^{\prime}$ have the same parity, then the number of squares in $\mathcal{T}(\lambda, v)$ is even. This implies that the parity of the number of squares in $\mathcal{T}(\lambda, v)$ of even hook length coincides with the parity of the number of squares in $\mathcal{T}(\lambda, v)$ of odd hook length. Similarly, for the case when $\lambda_{i}$ and $\lambda_{j}^{\prime}$ have different parities, it can be shown that the number of squares in $\mathcal{T}(\lambda, v)$ of even hook length is of opposite parity to the number of squares in $\mathcal{T}(\lambda, v)$ of odd hook length. Hence we arrive at (3.26).

By the inductive hypothesis, we see that $\mathcal{O}\left(\lambda^{-}\right) \equiv \mathcal{O}\left(\left(\lambda^{-}\right)^{\prime}\right)(\bmod 4)$ if and only if $H_{e}\left(\lambda^{-}\right)$is even. For any inner corner $v=(i, j)$ of $\lambda$, if $\lambda_{i} \equiv \lambda_{j}^{\prime}(\bmod 2)$, then $\mathcal{O}(\lambda) \equiv \mathcal{O}\left(\lambda^{\prime}\right)$ $(\bmod 4)$ if and only if $\mathcal{O}\left(\lambda^{-}\right) \equiv \mathcal{O}\left(\left(\lambda^{-}\right)^{\prime}\right)(\bmod 4)$. By $(3.26)$, we find that in this case, $H_{e}(\lambda)$ and $H_{e}\left(\lambda^{-}\right)$have the same parity. Thus the assertion holds for any partition $\lambda$ of

$n+1$. The case that $\lambda_{i} \not \equiv \lambda_{j}^{\prime}(\bmod 2)$ can be justified in the same manner. This completes the proof.

From Theorem 3.1, we obtain a combinatorial interpretation for Stanley's partition function $t(n)$, which can be recast as a combinatorial interpretation for $u(n)$.

Theorem 3.2 The partition function $t(n)$ is equal to the number of partitions of $n$ with an even number of even hooks, and the partition function $u(n)$ is equal to the number of partitions of $n$ with an odd number of even hooks. 
Combining Theorem 2.1 and Theorem 3.2, we have the following parity property.

Corollary 3.3 For any positive integer $n$, the number of partitions of $n$ with an odd number of even hooks is always even.

Since $f(n)=t(n)-u(n)$, from Theorem 3.2 we see that $f(n)$ can be interpreted as a signed counting of partitions of $n$ with respect to the number of even hooks, as stated below.

Corollary 3.4 The function $f(n)$ equals the number of partitions of $n$ with an even number of even hooks minus the number of partitions of $n$ with an odd number of even hooks.

To conclude, we remark that Corollary 3.4 can also be deduced from an identity of Han [6, Corollary 5.2$]$ by setting $t=2$.

Acknowledgments. This work was supported by the 973 Project, the PCSIRT Project of the Ministry of Education, and the National Science Foundation of China.

\section{References}

[1] G.E. Andrews, The Theory of Partitions, Addison-Wesley, 1976.

[2] G.E. Andrews, On a partition function of Richard Stanley, Electron. J. Combin. 11 (2) (2004) R1.

[3] G.E. Andrews and K. Eriksson, Integer Partitions, Cambridge Unversity Press, 2004.

[4] B.C. Berndt, Number Theory in the Spirit of Ramanujan, Amer. Math. Soc., Providence, 2004.

[5] G. Gasper and M. Rahman, Basic Hypergeometric Series, Cambridge University Press, Cambridge, 1990.

[6] G.N. Han, The Nekrasov-Okounkov hook length formula: refinement, elementary proof, extension and applications, Annales de l'Institut Fourier, 2009, 29 pages.

[7] R.P. Stanley, Enumerative Combinatorics, Vol. 2, Cambridge University Press, Cambridge, 1999.

[8] R.P. Stanley, Problem 10969, Amer. Math. Monthly 109 (2002) 760.

[9] R.P. Stanley, Some remarks on sign-balanced and maj-balanced posets, Adv. Appl. Math. 34 (2005) 880-902. 